\title{
Micro-channels fabrication through pulsed Nd:YAG laser on Ti6Al4V
}

\author{
Abhilasha Singh ${ }^{1}$, Divyansh Patel ${ }^{1}$, J. Ramkumar ${ }^{*}$ and K. Balani ${ }^{2}$ \\ ${ }^{1}$ Department of Mechanical Engineering, ${ }^{2}$ Department of Material Science and Engineering, \\ Indian Institute of Technology Kanpur, Kanpur -208016, India \\ *Email: jrkumar@iitk.ac.in
}

Fabrication of micro-channels is on great demand due to many applications in the area of bioengineering, microfluidic, heat exchangers and applications in investigating the flow rate performance of micro fluid channels (liquid dosing). Micro channel are considered as integral component for the purpose of transferring fluids to various components after the rise in miniaturization [1]. Although, there are several existing techniques for creating micro size features such as electrochemical micro-machining, electric discharge micro-machining, focused ion beam, chemical etching, lithography etc., which are time consuming and involves multiple steps. But, laser micro-machining dominates all other micro-machining processes available, when compared in the aspects of economy, accuracy and processing time. Some of the previous studies show the creation of micro-channels using laser micromachining $[2,3]$. In relation to this, experimental investigations are performed for carrying out the parametric study on titanium alloy surface, through solid state pulsed ytterbium fiber laser. Micro-channels of different geometries are machined on titanium surface at a range of predetermined input parameters (speed, frequency and power) to explore the machining capability of the process on titanium alloy. Design of experiment is utilized for obtaining sets of experiment for straight micro-channels with minimum possible width and an appropriate depth which can serve as passage to flow of a liquid. Micro-channels having width of $\approx 50 \mu \mathrm{m}$ and depth of $\approx 50 \mu \mathrm{m}$ are achieved at $100 \%$ power (maximum- 30 Watt), 30\% speed (maximum- 600 $\mathrm{mm} / \mathrm{s}$ ) and $30 \%$ frequency (maximum $-30 \mathrm{KHz}$ ). Using these parameters curved micro-channels and micromixtures are fabricated through ablating the titanium alloy on desired geometry. Figure 1 shows the effect of power and speed on width of the channel using one factor at a time approach. Increasing laser input power results increased width of channel (as shown in figure 1(a)) due to higher material removal rate (MRR).
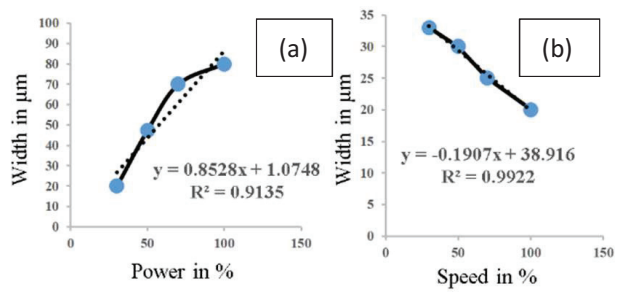

Figure 1: Graph to show the change in micro channels width with (a) power (constant speed and frequency), (b) speed (constant power and frequency)
Figure 1(b) shows that, as relative speed between laser head and workpiece is increased, width of the channel decreases. At low traverse speed, MRR is high due greater time interaction with the workpiece. It is observed that width and depth of the micro-channels increases with increase in machining power and decrease with machining speed. Figure 2(a) shows the micro-channel having width of $51.84 \mu \mathrm{m}$, made at high power $(100 \%)$ and constant speed $(30 \%)$ and frequency (30\%). Figure 2(b) shows the image of serpentine micro fluidic mixture having channel width of $70 \mu \mathrm{m}$. Figure 2(c) shows a magnified optical microscopy image of serpentine channels of micro mixture. Curved microchannel of $100 \mu \mathrm{m}$ width having radius of $500 \mu \mathrm{m}$ is shown in figure $2(d)$.

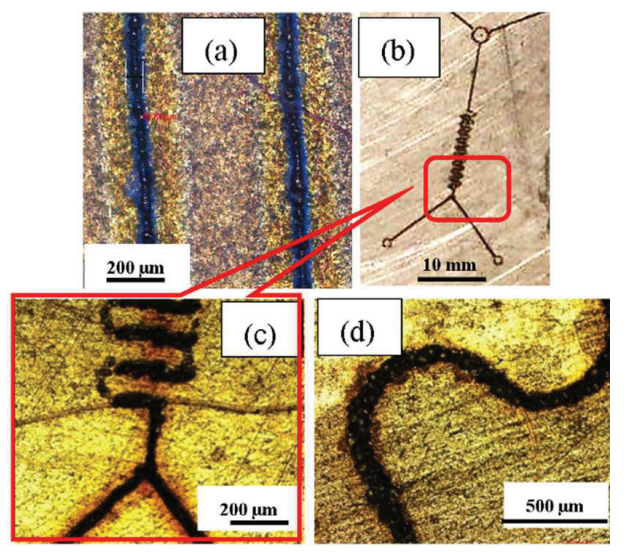

Figure 2: (a) Channel machined at high power and low speed on titanium surface. (b) Image of fluidic mixer (c) Optical image of serpentine area of micro fluidic mixture (d) Curved channel

Visual observation of the micro-features shows that the study need further investigation to improve the quality of the edges of the microchannels. Edge roughness of channels produced is high due to melting and resolidification of metal during laser-workpiece interaction. The future scope of research in this direction is: a hybrid laser micro-machining process is need to be designed by incorporating an appropriate finishing technique in it, to finish the micro-features during or just after the machining.

\section{References}

1. M. Nadasan, A. Manea, U.P.B. Sci. Bull (2009) 125-134. 2. S. Darwish, N. Ahmed, A. M. Alahmari, N. A. Mufti, Int J Adv Manuf Technol (2015)1-17.

3. N. Ahmed, S. Darwish, A. Alahmari Muhammad, A. Shar, Int J Adv Manuf Technol (2015) 1-14. 